\title{
LINEAR COMBINATIONS OF COMPOSITION OPERATORS ON THE BLOCH SPACES
}

\author{
TAKUYA HOSOKAWA, PEKKA J. NIEMINEN, AND SHÛICHI OHNO
}

\begin{abstract}
We characterize the compactness of linear combinations of analytic composition operators on the Bloch space. We also study their boundedness and compactness on the little Bloch space.
\end{abstract}

\section{INTRODUCTION}

Let $\mathbb{D}$ be the open unit disc of the complex plane $\mathbb{C}$ and let $\varphi$ be an analytic map which takes $\mathbb{D}$ into itself (often called an analytic self-map of $\mathbb{D})$. The composition operator induced by $\varphi$ is the linear operator $C_{\varphi}$ defined by $C_{\varphi} f=f \circ \varphi$ for any analytic function $f: \mathbb{D} \rightarrow \mathbb{C}$. The properties of such operators on various function spaces have been studied extensively during the past few decades (see [2] for an overview as of the mid-1990s).

The classical Bloch space, denoted by $\mathcal{B}$, consists of all analytic functions $f: \mathbb{D} \rightarrow \mathbb{C}$ satisfying

$$
\|f\|=\sup \left\{\left(1-|z|^{2}\right)\left|f^{\prime}(z)\right|: z \in \mathbb{D}\right\}<\infty .
$$

The norm $\|f\|_{\mathcal{B}}=|f(0)|+\|f\|$ makes $\mathcal{B}$ a Banach space. It is a simple consequence of the Schwarz-Pick inequality that any composition operator $C_{\varphi}$ takes the Bloch space into itself, thus defining a bounded operator on $\mathcal{B}$.

In this paper we will study linear combinations of composition operators, that is, operators of the form

$$
T=\lambda_{1} C_{\varphi_{1}}+\cdots+\lambda_{N} C_{\varphi_{N}}
$$

where $\lambda_{1}, \ldots, \lambda_{N}$ are nonzero complex scalars and $\varphi_{1}, \ldots, \varphi_{N}$ are analytic self-maps of $\mathbb{D}$. Our main goal is to give a function-theoretic characterization in terms of the coefficients $\lambda_{i}$ and maps $\varphi_{i}$ for when $T$ is compact on $\mathcal{B}$ (i.e. maps bounded sets into relatively compact ones). We will also characterize the boundedness and compactness of $T$ on the little Bloch space $\mathcal{B}_{0}$ consisting of those functions $f \in \mathcal{B}$ for which $\left(1-|z|^{2}\right)\left|f^{\prime}(z)\right| \rightarrow 0$ as $|z| \rightarrow 1$.

Date: 18 December 2008.

1991 Mathematics Subject Classification. Primary 47B33; Secondary 30D45, 47B07.

Key words and phrases. composition operator, compactness, Bloch space.

The first author is supported by the Korean Research Foundation Grant funded by Korean Government (KRF-2008-314-C00012). The second author was supported by the Academy of Finland, projects 118422 and 126420, and the Government of Aragón (Spain), project DGA E-64. The third author is partially supported by Grant-in-Aid for Scientific Research (no. 20540185), Japan Society for the Promotion of Science. 
Our work can be seen as a natural extension of previous research concerning compactness properties of a single composition operator or the difference of a pair of them acting on the Bloch spaces. Indeed, the analysis of composition operators in the context of Bloch spaces was initiated in 1995 by Madigan and Matheson [12], who characterized the compactness of $C_{\varphi}$ on $\mathcal{B}$ and $\mathcal{B}_{0}$ in terms of the hyperbolic derivative of $\varphi$ (see Section 2). More recently the authors of the present paper have investigated compact differences of composition operators on these spaces $[8,9,13]$, and the first author has also considered the more general case of weighted composition operators [7]. The study of differences is directly related to and motivated by another topic of considerable interest - that of describing the topological structure of the set of all composition operators acting on $\mathcal{B}$ or $\mathcal{B}_{0}$ (see in particular [8]).

Another, perhaps more interesting, aspect of studying compact linear combinations of the form (1.1) is the following. On any reasonable function space, including $\mathcal{B}$ and $\mathcal{B}_{0}$, the operator $T$ vanishes identically only in the case that the maps $\varphi_{i}$ all agree and $\lambda_{1}+\cdots+\lambda_{N}=0$ (see e.g. Exercise 1.1.10 in [2]). Thus there are no nontrivial linear relations in the set of composition operators. However, it is usually possible to find plenty of examples where $T$ becomes compact - or equivalently, vanishes in the corresponding Calkin algebra of operators (i.e. in the quotient algebra of bounded operators by the compact ones). Thus, from the Calkin algebra viewpoint, there are interesting linear relations among composition operators, and characterizing when $T$ is compact amounts to describing these.

The compactness of (1.1)-type linear combinations has recently been studied in a few other settings. Gorkin and Mortini [5] considered the norms and the essential norms (i.e. distances from the compact operators) of linear combinations of endomorphisms on general uniform algebras. Here endomorphisms can be represented as composition operators. In the particular case of $H^{\infty}$, the space of bounded analytic functions on $\mathbb{D}$, Izuchi and the third author [10] obtained more explicit results and gave a complete characterization for the compactness of $T$. In the results of these papers, the pseudo-hyperbolic distances between different members $\varphi_{i}$ and $\varphi_{j}$ of the linear combination have played a central role. In another direction, Kriete and Moorhouse [11] have initiated the study of linear combinations of composition operators on the Hardy space $H^{2}$ and weighted Dirichlet spaces.

Our main result is Theorem 3.2, which characterizes the compactness of $T$ on the Bloch space $\mathcal{B}$. As is perhaps to be expected, the characterizing condition is of the form

$$
\sum_{i \in I} \lambda_{i}=0 \quad \text { for certain sets } I \subset\{1, \ldots, N\} .
$$

These sets $I$ will be determined by the boundary behaviour of the hyperbolic derivatives of the maps $\varphi_{i}$ and the pseudo-hyperbolic distances between pairs of them; it will actually turn out that a couple of apparently different 
but equivalent formulations are possible. The results obtained and ideas employed in the proofs are partially similar to those in the $H^{\infty}$ case [10]; however, the important role played by the hyperbolic derivatives will give rise to major additional complications. For this reason we need to borrow some techniques from [13] in a refined form.

The paper is organized as follows. In Section 2 we collect some preliminaries on hyperbolic distances and derivatives and we also review the basics of composition operators acting on the Bloch spaces. In Section 3 we state our main result concerning compact linear combinations of composition operators on $\mathcal{B}$ and give a related example. The main result is proved in Section 4 . Finally, in Section 5 we consider the boundedness and compactness of such linear combinations on the little Bloch space $\mathcal{B}_{0}$.

\section{Preliminaries}

In this section we review some preliminaries on hyperbolic metrics and derivatives and on composition operators in the Bloch space context. After recalling the basic concepts, we collect a few useful lemmas that will be needed later on. This material ought to be well known to experts in the field, and the reader may wish to skip this section on first reading and consult it later as the need arises.

2.1. Hyperbolic metrics and derivatives. For two points $z, w \in \mathbb{D}$, the pseudo-hyperbolic distance is given by $\rho(z, w)=|z-w| /|1-\bar{w} z|$. The hyperbolic distance between $z$ and $w$ is then

$$
\inf _{\gamma} \int_{\gamma} \frac{|d \zeta|}{1-|\zeta|^{2}}=\frac{1}{2} \log \frac{1+\rho(z, w)}{1-\rho(z, w)}
$$

where the infimum is taken over all rectifiable $\operatorname{arcs} \gamma$ joining $z$ and $w$ in $\mathbb{D}$.

For any analytic $\varphi: \mathbb{D} \rightarrow \mathbb{D}$, we define

$$
\varphi^{\#}(z)=\frac{1-|z|^{2}}{1-|\varphi(z)|^{2}} \varphi^{\prime}(z) .
$$

Then $\varphi^{\#}$ is the hyperbolic derivative of $\varphi$ in the sense that

$$
\left|\varphi^{\#}(z)\right|=\lim _{w \rightarrow z} \frac{\rho(\varphi(z), \varphi(w))}{\rho(z, w)} .
$$

By the invariant form of the Schwarz lemma, $\varphi$ is always a contraction with respect to $\rho$. Equivalently, $\left|\varphi^{\#}(z)\right| \leq 1$ for all $z \in \mathbb{D}$, which is the content of the classical Schwarz-Pick inequality. (See e.g. [3, Section I.1].)

2.2. Bloch spaces. Recall that the Bloch space $\mathcal{B}$ consists of all functions $f$ analytic in $\mathbb{D}$ and satisfying $\|f\|=\sup \left\{\left(1-|z|^{2}\right)\left|f^{\prime}(z)\right|: z \in \mathbb{D}\right\}<\infty$ (see Introduction). There are several other ways of describing Bloch functions. For instance, in view of the definition of the hyperbolic metric above, an analytic function $f: \mathbb{D} \rightarrow \mathbb{C}$ belongs to $\mathcal{B}$ if and only if it is Lipschitzcontinuous from the hyperbolic metric into the Euclidean one, $\|f\|$ being 
the corresponding Lipschitz-constant. It is well known that the space $H^{\infty}$ of bounded analytic functions on $\mathbb{D}$ is continuously embedded in $\mathcal{B}$.

The little Bloch space $\mathcal{B}_{0}$ was defined as the subspace of those $f \in \mathcal{B}$ for which $\left(1-|z|^{2}\right)\left|f^{\prime}(z)\right| \rightarrow 0$ as $|z| \rightarrow 1$. Equivalently, $\mathcal{B}_{0}$ is the closure of analytic polynomials in $\mathcal{B}$. Not all bounded analytic functions belong to $\mathcal{B}_{0}$; however, there are also unbounded functions in $\mathcal{B}_{0}$ and hence in $\mathcal{B}$.

(A classic reference on Bloch spaces is [1]. See also the book [14].)

2.3. Composition operators on $\mathcal{B}$ and $\mathcal{B}_{0}$. The fact that every analytic map $\varphi: \mathbb{D} \rightarrow \mathbb{D}$ induces a bounded composition operator on $\mathcal{B}$ is seen by the chain rule. Indeed, for $f \in \mathcal{B}$ we have the identity

$$
\left(1-|z|^{2}\right)\left|\left(C_{\varphi} f\right)^{\prime}(z)\right|=\left|\varphi^{\#}(z)\right| \cdot\left(1-|\varphi(z)|^{2}\right)\left|f^{\prime}(\varphi(z))\right|,
$$

which together with the Schwarz-Pick inequality shows that $\left\|C_{\varphi} f\right\| \leq\|f\|$ and hence $C_{\varphi} f \in \mathcal{B}$. One may then apply the closed graph theorem or a simple function-theoretic estimate on $|f(\varphi(0))|$ to conclude that $C_{\varphi}: \mathcal{B} \rightarrow \mathcal{B}$ is bounded. This was observed by Madigan and Matheson [12], who then proved that

$$
C_{\varphi} \text { is compact on } \mathcal{B} \text { if and only if } \varphi^{\#}(z) \rightarrow 0 \text { as }|\varphi(z)| \rightarrow 1 \text {. }
$$

In order for $C_{\varphi}$ to act boundedly on $\mathcal{B}_{0}$ (that is, take $\mathcal{B}_{0}$ into itself), an obvious necessary condition is that $\varphi \in \mathcal{B}_{0}$, since $\varphi=C_{\varphi} z$. In [12] it was noticed that this condition is also sufficient. Furthermore, in this case the compactness criterion can be expressed in the apparently stronger form that $\varphi^{\#}(z) \rightarrow 0$ as $|z| \rightarrow 1$.

We proceed to state a few lemmas pertaining to the continuity properties of Bloch functions and hyperbolic derivatives. In these lemmas $c$ denotes a universal positive numeric constant whose value is unimportant and may differ from one occurrence to another. The proof of the first lemma is omitted; it can be found in e.g. [6, Lemma 5.1] or [9].

2.4. Lemma. For $f \in \mathcal{B}$ and $z, w \in \mathbb{D}$,

$$
\left|\left(1-|z|^{2}\right) f^{\prime}(z)-\left(1-|w|^{2}\right) f^{\prime}(w)\right| \leq c\|f\| \rho(z, w) .
$$

The next lemma is similar in spirit and observes that the hyperbolic derivative of an analytic self-map of the disc is always Lipschitz-continuous from the pseudo-hyperbolic metric to the Euclidean metric. This lemma is a special case of $[4$, Theorem 6]. Since the proof given in [4] is quite long and technical, we include a more straightforward argument for the present special case, based on the preceding lemma.

For $w \in \mathbb{D}$ we use $\alpha_{w}$ to denote the standard self-inverse automorphism of $\mathbb{D}$ defined by $\alpha_{w}(z)=(w-z) /(1-\bar{w} z)$.

2.5. Lemma. Let $\varphi: \mathbb{D} \rightarrow \mathbb{D}$ be analytic. Then for all $z, w \in \mathbb{D}$,

$$
\left|\varphi^{\#}(z)-\varphi^{\#}(w)\right| \leq c \rho(z, w) .
$$


Proof. We first observe that the following inequality holds for all $z, w \in \mathbb{D}$ :

$$
\left|\frac{\left(1-|z|^{2}\right)\left(1-|w|^{2}\right)}{(1-\bar{w} z)^{2}}-1\right| \leq c \rho(z, w) .
$$

One way to check this is to apply Lemma 2.4 to the function $f(\zeta)=(1-$ $\left.|w|^{2}\right) / \bar{w}(1-\bar{w} \zeta)$.

Now fix $w \in \mathbb{D}$ and consider the function $g_{w}=\alpha_{\varphi(w)} \circ \varphi$. Since $g_{w}$ is bounded in modulus by 1 , we have $\left\|g_{w}\right\| \leq c$. Routine computations show that

$$
-\left(1-|z|^{2}\right) g_{w}^{\prime}(z)=\frac{\left(1-|\varphi(z)|^{2}\right)\left(1-|\varphi(w)|^{2}\right)}{(1-\overline{\varphi(w)} \varphi(z))^{2}} \varphi^{\#}(z)
$$

and

$$
-\left(1-|w|^{2}\right) g_{w}^{\prime}(w)=\varphi^{\#}(w) .
$$

By Lemma 2.4, the difference of quantities (2.2) and (2.3) is bounded in modulus by $c \rho(z, w)$. In addition, by $(2.1)$ and the invariant Schwarz lemma,

$$
\left|\frac{\left(1-|\varphi(z)|^{2}\right)\left(1-|\varphi(w)|^{2}\right)}{(1-\overline{\varphi(w)} \varphi(z))^{2}}-1\right| \leq c \rho(\varphi(z), \varphi(w)) \leq c \rho(z, w) .
$$

The lemma follows from these estimates by a simple application of the triangle inequality (also recall that $\left|\varphi^{\#}(z)\right| \leq 1$ ).

Our final lemma shows that hyperbolic derivatives indeed work as derivatives should in describing the local behaviour of the associated self-maps; instead of the Euclidean metric we are just considering the hyperbolic (or pseudo-hyperbolic) scale. In particular, if the values of two self-maps $\varphi$ and $\psi$ agree at some point $z_{0} \in \mathbb{D}$ but the hyperbolic derivatives are unequal, then the values of $\varphi$ and $\psi$ get uniformly hyperbolically separated in a hyperbolic vicinity of $z_{0}$.

2.6. Lemma. There are constants $c_{1}, c_{2}, c_{3}>0$ with the following property: If $\varphi, \psi$ are analytic self-maps of $\mathbb{D}$ such that $\left|\varphi^{\#}\left(z_{0}\right)-\psi^{\#}\left(z_{0}\right)\right| \geq$ a for some $z_{0} \in \mathbb{D}$ and $a>0$, then

$$
\rho(\varphi(z), \psi(z)) \geq c_{1} a \rho\left(z, z_{0}\right)-c_{2} \rho\left(\varphi\left(z_{0}\right), \psi\left(z_{0}\right)\right)
$$

whenever $\rho\left(z, z_{0}\right) \leq c_{3} a$.

Proof. Define $\varphi_{0}=\alpha_{\varphi\left(z_{0}\right)} \circ \varphi \circ \alpha_{z_{0}}$ and $\psi_{0}$ similarly in terms of $\psi$. Then $\varphi_{0}$ and $\psi_{0}$ both fix the origin, $\varphi_{0}^{\prime}(0)=\varphi^{\#}\left(z_{0}\right)$ and $\psi_{0}^{\prime}(0)=\psi^{\#}\left(z_{0}\right)$. We recall that the second derivative of any analytic self-map of $\mathbb{D}$ is bounded in the disc $|\zeta| \leq \frac{1}{2}$ by a universal constant. Therefore, for these values of $\zeta$, Taylor's formula yields an estimate of the form

$$
\left|\varphi_{0}(\zeta)-\psi_{0}(\zeta)\right| \geq\left|\varphi_{0}^{\prime}(0)-\psi_{0}^{\prime}(0)\right||\zeta|-c|\zeta|^{2} \geq(a-c|\zeta|)|\zeta| .
$$


Put $c_{3}=\min \left(\frac{1}{4}, \frac{1}{2 c}\right)$. Then, for $|\zeta| \leq c_{3} a$ we have $|\zeta| \leq \min \left(\frac{1}{2}, \frac{a}{2 c}\right)$ (since $a \leq 2$ by the Schwarz-Pick inequality) and hence $\left|\varphi_{0}(\zeta)-\psi_{0}(\zeta)\right| \geq \frac{1}{2} a|\zeta|$. On denoting $z=\alpha_{z_{0}}(\zeta)$, this inequality means that

$$
\left|\alpha_{\varphi\left(z_{0}\right)}(\varphi(z))-\alpha_{\psi\left(z_{0}\right)}(\psi(z))\right| \geq \frac{1}{2} a \rho\left(z, z_{0}\right)
$$

whenever $\rho\left(z, z_{0}\right) \leq c_{3} a$. The rest now follows from the general fact that for all $u, u^{\prime}, v, v^{\prime} \in \mathbb{D}$,

$$
\left|\alpha_{v}(u)-\alpha_{v^{\prime}}\left(u^{\prime}\right)\right| \leq 2 \rho\left(u, u^{\prime}\right)+c \rho\left(v, v^{\prime}\right) .
$$

We leave the verification of this to the reader (or see [13, Lemma 3.4]).

\section{Compactness on the Bloch space}

In this section we characterize compact linear combinations of composition operators on the Bloch space $\mathcal{B}$. We let $\varphi_{1}, \ldots, \varphi_{N}$ be any analytic self-maps of the unit disc and consider an operator of the form

$$
T=\sum_{i=1}^{N} \lambda_{i} C_{\varphi_{i}}
$$

where $\lambda_{1}, \ldots, \lambda_{N}$ are nonzero complex scalars. This notation will remain fixed throughout this section. We also write

$$
\rho_{i j}(z)=\rho\left(\varphi_{i}(z), \varphi_{j}(z)\right)
$$

for the pseudo-hyperbolic distance between $\varphi_{i}(z)$ and $\varphi_{j}(z)$.

It will be convenient to formulate our results in terms of suitable test sequences and associated partitions of the index set $\{1, \ldots, N\}$. We start by defining these.

3.1. Test sequences and partitions. Let $\Delta$ denote the collection of all sequences $\left\{z_{n}\right\}$ in $\mathbb{D}$ converging to some point of $\partial \mathbb{D}$ such that also the sequences $\left\{\varphi_{i}\left(z_{n}\right)\right\},\left\{\varphi_{i}^{\#}\left(z_{n}\right)\right\}$ and $\left\{\rho_{i j}\left(z_{n}\right)\right\}$ converge for all $i, j=1, \ldots, N$. Given a sequence $\left\{z_{n}\right\} \in \Delta$ and an index $j=1, \ldots, N$, define

$$
\begin{gathered}
I\left\{z_{n}\right\}=\left\{i:\left|\varphi_{i}\left(z_{n}\right)\right| \rightarrow 1\right\}, \\
I_{j}\left\{z_{n}\right\}=\left\{i: \rho_{i j}\left(z_{n}\right) \rightarrow 0\right\}, \\
I_{j}^{*}\left\{z_{n}\right\}=I_{j}\left\{z_{n}\right\} \cap\left\{i: \varphi_{i}^{\#}\left(z_{n}\right) \nrightarrow 0\right\}, \\
I_{j}^{\#}\left\{z_{n}\right\}=I_{j}\left\{z_{n}\right\} \cap\left\{i: \lim \varphi_{i}^{\#}\left(z_{n}\right)=\lim \varphi_{j}^{\#}\left(z_{n}\right)\right\} .
\end{gathered}
$$

All limits here are to be understood as $n \rightarrow \infty$.

Note that every sequence $\left\{z_{n}\right\}$ in $\mathbb{D}$ with $\left|z_{n}\right| \rightarrow 1$ has subsequences belonging to $\Delta$. As we will see, given a sequence $\left\{z_{n}\right\} \in \Delta$, only the indices in the set $I\left\{z_{n}\right\}$ will play a role in the compactness characterization of $T$ on $\mathcal{B}$. Furthermore, the sets $I_{j}\left\{z_{n}\right\}$ induce a natural partition of $I\left\{z_{n}\right\}$ (as well as of $\{1, \ldots, N\}$ ), which can be further refined by the sets $I_{j}^{\#}\left\{z_{n}\right\}$. For instance, for each $j \in I\left\{z_{n}\right\}$ we clearly have $j \in I_{j}\left\{z_{n}\right\} \subset I\left\{z_{n}\right\}$, and for 
different values of $j$ the sets $I_{j}\left\{z_{n}\right\}$ are either the same or disjoint. Thus, using ' + ' to denote disjoint unions, we may write

$$
I\left\{z_{n}\right\}=I_{j_{1}}\left\{z_{n}\right\}+\cdots+I_{j_{p}}\left\{z_{n}\right\}
$$

for some $j_{1}, \ldots, j_{p} \in I\left\{z_{n}\right\}$. Similarly, for each $j=1, \ldots, N$,

$$
I_{j}\left\{z_{n}\right\}=I_{k_{1}}^{\#}\left\{z_{n}\right\}+\cdots+I_{k_{q}}^{\#}\left\{z_{n}\right\},
$$

where $k_{1}, \ldots, k_{q} \in I_{j}\left\{z_{n}\right\}$.

The following theorem is our main result.

3.2. Theorem. The following conditions are equivalent:

(1) The operator $T$ of (3.1) is compact.

(2) $\sum_{i \in I_{j}\left\{z_{n}\right\}} \lambda_{i} \varphi_{i}^{\#}\left(z_{n}\right) \rightarrow 0$ as $n \rightarrow \infty$ for all $\left\{z_{n}\right\} \in \Delta, j \in I\left\{z_{n}\right\}$.

(3) $\sum_{i \in I_{j}^{*}\left\{z_{n}\right\}} \lambda_{i}=0$ for all $\left\{z_{n}\right\} \in \Delta, j \in I\left\{z_{n}\right\}$.

(4) $\sum_{i \in I_{j}^{\#}\left\{z_{n}\right\}} \lambda_{i}=0$ for all $\left\{z_{n}\right\} \in \Delta, j \in I\left\{z_{n}\right\}$ with $\varphi_{j}^{\#}\left(z_{n}\right) \not \rightarrow 0$.

The proof of Theorem 3.2 is given in the next section. Let us however make a few comments. As regards the necessity of the stated conditions, (2) is the easiest to prove while establishing (3) and (4) requires more careful analysis of the behaviour of hyperbolic derivatives, based on the lemmas presented in Section 2. In the other direction, (4) is the seemingly strongest of the conditions in the sense that it readily implies (2) and (3) for any fixed $\left\{z_{n}\right\}$.

3.3. Remark. The reader may wonder if the natural condition

$$
\sum_{i \in I_{j}\left\{z_{n}\right\}} \lambda_{i}=0 \text { for all }\left\{z_{n}\right\} \in \Delta, j \in I\left\{z_{n}\right\}
$$

could be added to the list above. It is indeed sufficient for the compactness of $T$. While not completely obvious from the statement of the theorem, this can be seen by examining the proof in the next section (implication (3) $\Rightarrow$ (4)). However, (3.2) fails to be necessary already for the case $N=1$ of one composition operator: any compact $C_{\varphi}$ with $\|\varphi\|_{\infty}=1$ provides a counterexample. In fact, (3.2) is precisely the characterization obtained in [10] for the compactness of $T$ on the space $H^{\infty}$ of bounded analytic functions on $\mathbb{D}$.

In the special case of a single composition operator we have $N=1, \lambda_{1}=1$, and Theorem 3.2 trivially reduces to the Madigan-Matheson compactness criterion saying that $\varphi_{1}^{\#}(z) \rightarrow 0$ as $\left|\varphi_{1}(z)\right| \rightarrow 1$ (see Section 2.2). For the case $N=2$ we have Corollary 3.4 below. Condition (1) of the corollary expresses the fact that basically only differences may exhibit interesting examples of compact linear combinations of two composition operators. Conditions 
(2) and (3) comprise the characterization of compact differences which was previously obtained in [13] (see also [8,9]).

3.4. Corollary. Let $\varphi_{1}$ and $\varphi_{2}$ be analytic self-maps of $\mathbb{D}$, and suppose that neither $C_{\varphi_{1}}$ nor $C_{\varphi_{2}}$ is compact on $\mathcal{B}$. Also let $\lambda_{1}, \lambda_{2} \in \mathbb{C} \backslash\{0\}$. Then $\lambda_{1} C_{\varphi_{1}}+\lambda_{2} C_{\varphi_{2}}$ is compact on $\mathcal{B}$ if and only if

(1) $\lambda_{1}+\lambda_{2}=0$,

and for each sequence $\left\{z_{n}\right\} \in \Delta$,

(2) if $\left|\varphi_{1}\left(z_{n}\right)\right| \rightarrow 1$, then $\varphi_{1}^{\#}\left(z_{n}\right) \rightarrow 0$ or $\rho_{12}\left(z_{n}\right) \rightarrow 0$,

(3) if $\left|\varphi_{2}\left(z_{n}\right)\right| \rightarrow 1$, then $\varphi_{2}^{\#}\left(z_{n}\right) \rightarrow 0$ or $\rho_{12}\left(z_{n}\right) \rightarrow 0$.

Proof. Apply condition (3) of Theorem 3.2. Observe that the noncompactness of $C_{\varphi_{j}}$ guarantees the existence of a sequence $\left\{z_{n}\right\} \in \Delta$ such that $j \in I\left\{z_{n}\right\}$ and $\varphi_{j}^{\#}\left(z_{n}\right) \not \rightarrow 0$.

For $N \geq 3$ different types of compact linear combinations may occur. Here we give an example where no linear combination of only two operators is compact.

3.5. Example. Let $\sigma(z)=(1+z) /(1-z)$ and let

$$
\varphi_{1}(z)=\frac{\sqrt{\sigma(z)}-1}{\sqrt{\sigma(z)}+1}
$$

be the so-called lens map. Put $\tau(z)=z+t(1-z)^{2}$. Then $\tau$ is a self-map of $\mathbb{D}$ for small enough $t>0$. Define $\varphi_{2}(z)=\tau\left(\varphi_{1}(z)\right)$ and $\varphi_{3}(z)=-\tau\left(-\varphi_{1}(z)\right)$. Then $C_{\varphi_{1}}-C_{\varphi_{2}}-C_{\varphi_{3}}$ is compact on $\mathcal{B}$.

Proof. Note that all the maps $\varphi_{1}, \varphi_{2}$ and $\varphi_{3}$ admit a continuous extension to the closed unit disc. The map $\varphi_{1}$ fixes the points 1 and -1 , and at each of these points its image has a vertex with an angle $\frac{1}{2} \pi$. It is also easy to verify that $\left|\varphi_{1}^{\#}(z)\right| \rightarrow \frac{1}{2}$ as $z \rightarrow \pm 1$ (see [8]), but this information is not really needed here. In addition, we have $\varphi_{2}(1)=1$ and $\left|\varphi_{2}\left(e^{\mathrm{i} \theta}\right)\right|<1$ for $e^{\mathrm{i} \theta} \neq 1$, and similarly, $\varphi_{3}(-1)=-1$ and $\left|\varphi_{3}\left(e^{\mathrm{i} \theta}\right)\right|<1$ for $e^{\mathrm{i} \theta} \neq-1$. Thus it is sufficient to consider only those test sequences $\left\{z_{n}\right\}$ that converge either to 1 or to -1 .

We show that for any sequence $\left\{z_{n}\right\}$ converging to 1 we have $\rho_{12}\left(z_{n}\right) \rightarrow 0$. Then $I_{1}\left\{z_{n}\right\}=\{1,2\}$ since clearly $3 \notin I\left\{z_{n}\right\}$. Indeed, writing $\varphi_{1}\left(z_{n}\right)=$ $1+r_{n} e^{\mathrm{i} \theta_{n}}$ we obtain

$$
\begin{aligned}
\rho_{12}\left(z_{n}\right) & =\left|\frac{t\left(1-\varphi_{1}\left(z_{n}\right)\right)^{2}}{1-\left|\varphi_{1}\left(z_{n}\right)\right|^{2}-t \overline{\varphi_{1}\left(z_{n}\right)}\left(1-\varphi_{1}\left(z_{n}\right)\right)^{2}}\right| \\
& =\frac{t r_{n}}{\left|r_{n}+2 \cos \theta_{n}+t r_{n}\left(1+r_{n} e^{-\mathrm{i} \theta_{n}}\right) e^{2 \mathrm{i} \theta_{n}}\right|} .
\end{aligned}
$$

Since $\frac{3}{4} \pi \leq \theta_{n} \leq \frac{5}{4} \pi$ for all $n$ and $r_{n} \rightarrow 0$, we get $\rho_{12}\left(z_{n}\right) \rightarrow 0$ as $n \rightarrow \infty$. 
Similarly one may show that $I_{1}\left\{z_{n}\right\}=\{1,3\}$ for $\left\{z_{n}\right\}$ converging to -1 . The compactness of $T$ now follows from Theorem 3.2, or by the sufficient condition (3.2).

\section{Proof of Theorem 3.2}

The outline of the proof is the following. We first establish the equivalence of (1) and (2). This step is rather standard to anyone familiar with composition operators, and involves constructing suitable test functions for the necessity part. Then we proceed to show that each of (2) and (3) implies (4). This depends on a more delicate analysis of hyperbolic derivatives and related mapping properties of self-maps and we will utilize the lemmas given towards the end of Section 2. Finally we observe that (almost trivially) (4) implies both (2) and (3).

In establishing the equivalence of (1) and (2) we will make use of the following well-known principle: the operator $T$ is compact if and only if $\left\|T f_{n}\right\|_{\mathcal{B}} \rightarrow 0$ whenever $\left\{f_{n}\right\}$ is a bounded sequence in $\mathcal{B}$ such that $f_{n} \rightarrow 0$ pointwise. The main reasons for this are that composition operators preserve pointwise convergence, and that any bounded sequence in $\mathcal{B}$ is a normal family, thus containing subsequences that convergence pointwise (even uniformly on compact sets); cf. [2, Proposition 3.11].

4.1. (2) implies (1). We assume to the contrary that $T$ is noncompact. This implies that there exists a bounded sequence $\left\{f_{n}\right\}$ in $\mathcal{B}$ such that $f_{n} \rightarrow 0$ uniformly on compact sets but $\left\|T f_{n}\right\|_{\mathcal{B}}>C$ for all $n$, where $C>0$. Hence we can find points $z_{n} \in \mathbb{D}$ such that for large $n$,

$$
\begin{aligned}
C & <\left(1-\left|z_{n}\right|^{2}\right)\left|\left(T f_{n}\right)^{\prime}\left(z_{n}\right)\right| \\
& =\left(1-\left|z_{n}\right|^{2}\right)\left|\sum_{i=1}^{N} \lambda_{i} f_{n}^{\prime}\left(\varphi_{i}\left(z_{n}\right)\right) \varphi_{i}^{\prime}\left(z_{n}\right)\right| \\
& =\left|\sum_{i=1}^{N} \lambda_{i} \varphi_{i}^{\#}\left(z_{n}\right)\left(1-\left|\varphi_{i}\left(z_{n}\right)\right|^{2}\right) f_{n}^{\prime}\left(\varphi_{i}\left(z_{n}\right)\right)\right| .
\end{aligned}
$$

Passing to a subsequence we may assume that $\left\{z_{n}\right\} \in \Delta$. Since the derivatives $\varphi_{i}^{\#}$ are uniformly bounded and $f_{n}^{\prime} \rightarrow 0$ on compact sets, the preceding inequality remains true for large values of $n$ even if the sum is taken over indices $i \in I\left\{z_{n}\right\}$ only. We may further write $I\left\{z_{n}\right\}$ as the disjoint union $I\left\{z_{n}\right\}=\bigcup_{s=1}^{p} J_{s}$ where $J_{s}=I_{j_{s}}\left\{z_{n}\right\}$ for some $j_{s} \in I\left\{z_{n}\right\}$. Therefore, for some (henceforth fixed) $s$ and for sufficiently large $n$,

$$
\left|\sum_{i \in J_{s}} \lambda_{i} \varphi_{i}^{\#}\left(z_{n}\right)\left(1-\left|\varphi_{i}\left(z_{n}\right)\right|^{2}\right) f_{n}^{\prime}\left(\varphi_{i}\left(z_{n}\right)\right)\right|>\frac{C}{p} .
$$

Since $\left\{f_{n}\right\}$ is bounded in $\mathcal{B}$, the quantities $\left(1-\left|\varphi_{i}\left(z_{n}\right)\right|^{2}\right) f_{n}^{\prime}\left(\varphi_{i}\left(z_{n}\right)\right)$ stay bounded. Moreover, Lemma 2.4 ensures that for $i, j \in J_{s}$,

$$
\left|\left(1-\left|\varphi_{i}\left(z_{n}\right)\right|^{2}\right) f_{n}^{\prime}\left(\varphi_{i}\left(z_{n}\right)\right)-\left(1-\left|\varphi_{j}\left(z_{n}\right)\right|^{2}\right) f_{n}^{\prime}\left(\varphi_{j}\left(z_{n}\right)\right)\right| \leq c \rho_{i j}\left(z_{n}\right) \rightarrow 0
$$


as $n \rightarrow \infty$. These facts combine to show that

$$
\underset{n \rightarrow \infty}{\limsup }\left|\sum_{i \in J_{s}} \lambda_{i} \varphi_{i}^{\#}\left(z_{n}\right)\right|>0
$$

which is a contradiction and completes the proof.

4.2. (1) implies (2). Suppose that $\left\{z_{n}\right\} \in \Delta$ is a sequence for which $\left|\varphi_{1}\left(z_{n}\right)\right| \rightarrow 1$. We write $I=I\left\{z_{n}\right\}$ and $J=I_{1}\left\{z_{n}\right\}$. Our aim is to show that

$$
\lim _{n \rightarrow \infty} \sum_{i \in J} \lambda_{i} \varphi_{i}^{\#}\left(z_{n}\right)=0
$$

Let us recall the notation $\alpha_{w}(z)=(w-z) /(1-\bar{w} z)$ used for the disc automorphism that exchanges the points 0 and $w$. Let $\left\{f_{n}\right\}$ be the sequence of analytic functions defined on $\mathbb{D}$ by

$$
f_{n}(z)=\alpha_{\varphi_{1}\left(z_{n}\right)}(z) \prod_{i \in I \backslash J} \alpha_{\varphi_{i}\left(z_{n}\right)}(z)^{2}-\gamma_{n},
$$

where $\gamma_{n}=\varphi_{1}\left(z_{n}\right) \prod_{i \in I \backslash J} \varphi_{i}\left(z_{n}\right)^{2}$. Then $\left\{f_{n}\right\}$ is a bounded sequence in $H^{\infty}$ and hence in $\mathcal{B}$. The constants $\gamma_{n}$ are chosen such that $f_{n}(0)=0$ for each $n$. Moreover, since $\alpha_{w}-w \rightarrow 0$ uniformly on compact sets as $|w| \rightarrow 1$, it follows that $f_{n} \rightarrow 0$ uniformly on compacts. Hence $\left\|T f_{n}\right\|_{\mathcal{B}} \rightarrow 0$.

Next consider the estimate

$$
\begin{aligned}
\left\|T f_{n}\right\|_{\mathcal{B}} & \geq\left(1-\left|z_{n}\right|^{2}\right)\left|\left(T f_{n}\right)^{\prime}\left(z_{n}\right)\right| \\
& =\left|\sum_{i=1}^{N} \lambda_{i} \varphi_{i}^{\#}\left(z_{n}\right)\left(1-\left|\varphi_{i}\left(z_{n}\right)\right|^{2}\right) f_{n}^{\prime}\left(\varphi_{i}\left(z_{n}\right)\right)\right| .
\end{aligned}
$$

Since $f_{n}^{\prime} \rightarrow 0$ uniformly on compact sets, we conclude exactly as in the proof of the converse implication before that the terms corresponding to indices $i \notin I$ tend to zero as $n \rightarrow \infty$. For $i \in I \backslash J$ we note that the function $f_{n}$ has the property that $f_{n}^{\prime}\left(\varphi_{i}\left(z_{n}\right)\right)=0$. For $i \in J$ we have $\rho_{1 i}\left(z_{n}\right) \rightarrow 0$ so Lemma 2.4 again yields

$$
\left|\left(1-\left|\varphi_{i}\left(z_{n}\right)\right|^{2}\right) f_{n}^{\prime}\left(\varphi_{i}\left(z_{n}\right)\right)-\left(1-\left|\varphi_{1}\left(z_{n}\right)\right|^{2}\right) f_{n}^{\prime}\left(\varphi_{1}\left(z_{n}\right)\right)\right| \rightarrow 0
$$

as $n \rightarrow \infty$. Putting these facts together and recalling that $\left\|T f_{n}\right\|_{\mathcal{B}} \rightarrow 0$, we obtain from (4.2) that

$$
\lim _{n \rightarrow \infty}\left|\sum_{i \in J} \lambda_{i} \varphi_{i}^{\#}\left(z_{n}\right)\right|\left(1-\left|\varphi_{1}\left(z_{n}\right)\right|^{2}\right)\left|f_{n}^{\prime}\left(\varphi_{1}\left(z_{n}\right)\right)\right|=0 .
$$

Now consider the derivative $f_{n}^{\prime}\left(\varphi_{1}\left(z_{n}\right)\right)$. Since $\alpha_{\varphi_{1}\left(z_{n}\right)}\left(\varphi_{1}\left(z_{n}\right)\right)=0$, we see that when applying the product rule of differentiation to it, the only nonzero contribution comes from the term containing the factor $\alpha_{\varphi_{1}\left(z_{n}\right)}^{\prime}\left(\varphi_{1}\left(z_{n}\right)\right)$, which equals $-\left(1-\left|\varphi_{1}\left(z_{n}\right)\right|^{2}\right)^{-1}$. Hence

$$
\left(1-\left|\varphi_{1}\left(z_{n}\right)\right|^{2}\right)\left|f_{n}^{\prime}\left(\varphi_{1}\left(z_{n}\right)\right)\right|=\left|\prod_{i \in I \backslash J} \alpha_{\varphi_{i}\left(z_{n}\right)}\left(\varphi_{1}\left(z_{n}\right)\right)^{2}\right|=\prod_{i \in I \backslash J} \rho_{1 i}\left(z_{n}\right)^{2} .
$$


Since the limit of this is nonzero, the desired conclusion (4.1) follows.

In order to prove the necessity of (3) and (4), we first establish a lemma that enables us to move from partitions induced by $I_{j}^{\#}$-type sets back to partitions induced by $I_{j}$-type sets. The lemma is based on the following idea: It may easily happen that along some sequence $\left\{z_{n}\right\} \in \Delta$ we have $\rho_{i j}\left(z_{n}\right) \rightarrow 0$ but the limits of $\varphi_{i}^{\#}\left(z_{n}\right)$ and $\varphi_{j}^{\#}\left(z_{n}\right)$ are unequal, so that $i \in$ $I_{j}\left\{z_{n}\right\}$ but $i \notin I_{j}^{\#}\left\{z_{n}\right\}$. However, in such a situation Lemma 2.6 implies that a small perturbation to the points $z_{n}$ will separate the values of $\varphi_{i}$ and $\varphi_{j}$ hyperbolically, and thus split the corresponding $I_{j}$-equivalence class accordingly. This line of thought (in a more implicit form) was already used in [13] to analyse differences of composition operators on $\mathcal{B}$.

4.3. Lemma. Assume that $\left\{z_{n}\right\} \in \Delta$ and $j=1, \ldots, N$. There exists an $\epsilon_{0}>0$ with the following property: If $w_{n} \in \mathbb{D}$ are points with $\rho\left(z_{n}, w_{n}\right)=$ $\rho\left(z_{1}, w_{1}\right) \in\left(0, \epsilon_{0}\right)$ for all $n$, then $\left\{w_{n}\right\}$ has a subsequence $\left\{w_{n}^{\prime}\right\} \in \Delta$ such that

$$
I_{j}^{\#}\left\{z_{n}\right\}=I_{j_{1}}\left\{w_{n}^{\prime}\right\}+\cdots+I_{j_{p}}\left\{w_{n}^{\prime}\right\}
$$

for some $j_{1}, \ldots, j_{p}$. Here $j_{1}, \ldots, j_{p} \in I\left\{w_{n}^{\prime}\right\}$ provided that $j \in I\left\{z_{n}\right\}$.

Proof. Assume that $j=1$, and write $J=I_{1}\left\{z_{n}\right\}$ and $J^{\#}=I_{1}^{\#}\left\{z_{n}\right\}$. Then for some complex constant $d, \lim \varphi_{i}^{\#}\left(z_{n}\right)=d$ for all $i \in J^{\#} \operatorname{but} \lim \varphi_{i}^{\#}\left(z_{n}\right) \neq$ $d$ for all $i \in J \backslash J \#$. Now Lemma 2.6 implies that there exist $\epsilon_{1}>0$ and $c>0$ such that the following holds for sufficiently large $n$ : whenever $w \in \mathbb{D}$ is a point for which $\rho\left(w, z_{n}\right) \leq \epsilon_{1}$, then $\rho_{i i^{\prime}}(w) \geq c \rho\left(w, z_{n}\right)$ for $i \in J^{\#}$, $i^{\prime} \in J \backslash J^{\#}$.

On the other hand, for any $i \in J, i^{\prime} \in J^{c}$ (complement with respect to $\{1, \ldots, N\})$, we have $\lim \rho_{i i^{\prime}}\left(z_{n}\right)>0$. Thus if $\epsilon_{2}>0$ is small enough and $\rho\left(w_{n}, z_{n}\right) \leq \epsilon_{2}$ for all $n$, then $\liminf \rho_{i i^{\prime}}\left(w_{n}\right)>0$ for $i \in J, i^{\prime} \in J^{c}$ because each analytic self-map of $\mathbb{D}$ is contractive with respect to $\rho$.

Let $\epsilon_{0}=\min \left(\epsilon_{1}, \epsilon_{2}\right)$, and choose points $w_{n}$ with $\rho\left(w_{n}, z_{n}\right)=\rho\left(w_{1}, z_{1}\right) \in$ $\left(0, \epsilon_{0}\right)$. The previous observations combine to show that $\lim \inf \rho_{i i^{\prime}}\left(w_{n}\right)>0$ for $i \in J^{\#}, i^{\prime} \in\left(J^{\#}\right)^{c}$. Therefore $\left\{w_{n}\right\}$ has a subsequence $\left\{w_{n}^{\prime}\right\}$ in $\Delta$ and the set $J^{\#}$ consists of one or more sets of the form $I_{k}\left\{w_{n}^{\prime}\right\}$, as required. Clearly $\left|\varphi_{i}\left(w_{n}\right)\right| \rightarrow 1$ for $i \in J^{\#}$ provided that $\left|\varphi_{1}\left(z_{n}\right)\right| \rightarrow 1$, establishing the last claim.

4.4. (2) implies (4). We assume that $\left\{z_{n}\right\} \in \Delta$ and $j \in I\left\{z_{n}\right\}$ such that $\lim \varphi_{j}^{\#}\left(z_{n}\right) \neq 0$. If $\epsilon>0$ is small enough and $\rho\left(z_{n}, w_{n}\right)=\epsilon$ for all $n$, then Lemma 4.3 guarantees the existence of a subsequence $\left\{w_{n}^{\prime}\right\} \in \Delta$ such that

$$
I_{j}^{\#}\left\{z_{n}\right\}=I_{j_{1}}\left\{w_{n}^{\prime}\right\}+\cdots+I_{j_{p}}\left\{w_{n}^{\prime}\right\}
$$

where $j_{1}, \ldots, j_{p} \in I\left\{w_{n}^{\prime}\right\}$. Therefore our assumption shows that

$$
\lim _{n \rightarrow \infty} \sum_{i \in I_{j}^{\#}\left\{z_{n}\right\}} \lambda_{i} \varphi_{i}^{\#}\left(w_{n}^{\prime}\right)=0 .
$$


By Lemma 2.5 we have, for all $i$ and $n,\left|\varphi_{i}^{\#}\left(w_{n}\right)-\varphi_{i}^{\#}\left(z_{n}\right)\right| \leq c \epsilon$. Since $\epsilon$ can be made arbitrarily small and $\lim \varphi_{i}^{\#}\left(z_{n}\right)=\lim \varphi_{j}^{\#}\left(z_{n}\right) \neq 0$ for $i \in I_{j}^{\#}\left\{z_{n}\right\}$, we conclude that $\sum_{i \in I_{j}^{\#}\left\{z_{n}\right\}} \lambda_{i}=0$.

4.5. (3) implies (4). As above, assume that $\left\{z_{n}\right\} \in \Delta$ and $j \in I\left\{z_{n}\right\}$ such that the limit of $\varphi_{j}^{\#}\left(z_{n}\right)$ is nonzero. Once again, if $\epsilon>0$ is small enough, Lemma 4.3 shows that

$$
I_{j}^{\#}\left\{z_{n}\right\}=I_{j_{1}}\left\{w_{n}^{\prime}\right\}+\cdots+I_{j_{p}}\left\{w_{n}^{\prime}\right\}
$$

where $j_{1}, \ldots, j_{p} \in I\left\{w_{n}^{\prime}\right\}$ and $\left\{w_{n}^{\prime}\right\}$ is a subsequence of some $\left\{w_{n}\right\}$ with $\rho\left(z_{n}, w_{n}\right)=\epsilon$. By choosing a smaller $\epsilon$, if necessary, we may assume that the limit of $\varphi_{i}^{\#}\left(w_{n}^{\prime}\right)$ is nonzero for each $i \in I_{j}^{\#}\left\{z_{n}\right\}$; this follows by the continuity of the hyperbolic derivative (Lemma 2.5). Now our assumption ensures that $\sum_{i \in I_{j_{s}}\left\{w_{n}^{\prime}\right\}} \lambda_{i}=0$ for each $s=1, \ldots, p$. Hence $\sum_{i \in I_{j}^{\#}\left\{z_{n}\right\}} \lambda_{i}=0$.

4.6. (4) implies (2) and (3). Given $\left\{z_{n}\right\} \in \Delta$ and $j \in I\left\{z_{n}\right\}$, we write

$$
I_{j}\left\{z_{n}\right\}=I_{k_{1}}^{\#}\left\{z_{n}\right\}+\cdots+I_{k_{q}}^{\#}\left\{z_{n}\right\}
$$

with $k_{1}, \ldots, k_{q} \in I_{j}\left\{z_{n}\right\}$. Hence

$$
\sum_{i \in I_{j}\left\{z_{n}\right\}} \lambda_{i} \varphi_{i}^{\#}\left(z_{n}\right)=\sum_{s=1}^{q} \sum_{i \in I_{k_{s}}^{\#}\left\{z_{n}\right\}} \lambda_{i} \varphi_{i}^{\#}\left(z_{n}\right) .
$$

Since the limits of $\varphi_{i}^{\#}\left(z_{n}\right)$ agree for all terms in each of the inner sums, our assumption ensures that each inner sum (and hence the whole expression) tends to zero as $n \rightarrow \infty$. This gives (2). It is even easier to deduce (3); we leave this to the reader.

We close this section by a functional-analytic remark.

4.7. Remark. Apart from compactness, Theorem 3.2 also characterizes when the operator $T$ is weakly compact (i.e. takes bounded sets of $\mathcal{B}$ into sets that are relatively compact in the weak topology). To see this, one may modify the test functions $f_{n}$ constructed in Section 4.2 (implication $(1) \Rightarrow$ $(2)$ ) so that the sequence $\left\{f_{n}\right\}$ becomes equivalent with the standard basis of the sequence space $c_{0}$. This makes it possible to invoke the Dunford-Pettis property of $c_{0}$ and conclude that the operator $T$, now assumed only weakly compact, sends the sequence $\left\{f_{n}\right\}$ onto a norm-null sequence as required for the proof of condition (2). We omit the details and refer the reader to e.g. [13] where such an argument was carried out. 


\section{Boundedness and Compactness on the little Bloch space}

In this section we consider linear combinations of composition operators acting on the little Bloch space $\mathcal{B}_{0}$. As before, we let

$$
T=\sum_{i=1}^{N} \lambda_{i} C_{\varphi_{i}}
$$

where $\varphi_{1}, \ldots, \varphi_{N}$ are analytic self-maps of the unit disc and $\lambda_{1}, \ldots, \lambda_{N}$ are nonzero complex scalars.

As in the case of a single composition operator, we first need to examine the boundedness of $T$ on $\mathcal{B}_{0}$. Retaining the notation explained in Section 3.1, we introduce one more partition of our index set $\{1, \ldots, N\}$ : for $\left\{z_{n}\right\} \in \Delta$ and $j=1, \ldots, N$, let

$$
J_{j}\left\{z_{n}\right\}=\left\{i: \lim _{n \rightarrow \infty} \varphi_{i}\left(z_{n}\right)=\lim _{n \rightarrow \infty} \varphi_{j}\left(z_{n}\right)\right\} .
$$

Note that the partition induced by these sets is coarser than the one induced by the sets $I_{j}\left\{z_{n}\right\}$ because convergence in the pseudo-hyperbolic metric always implies convergence in the Euclidean metric.

5.1. Theorem. Consider the operator $T$ defined above. The following are equivalent:

(1) $T$ is bounded on $\mathcal{B}_{0}$, i.e. maps $\mathcal{B}_{0}$ into $\mathcal{B}_{0}$.

(2) $T z^{k} \in \mathcal{B}_{0}$ for $k=1, \ldots, N$.

(3) For $\left\{z_{n}\right\} \in \Delta$ and $j=1, \ldots, N$,

$$
\lim _{n \rightarrow \infty}\left(1-\left|z_{n}\right|^{2}\right) \sum_{i \in J_{j}\left\{z_{n}\right\}} \lambda_{i} \varphi_{i}^{\prime}\left(z_{n}\right)=0 .
$$

It is worth noting here that the convergence requirements placed on $\left\{z_{n}\right\} \in \Delta$ guarantee that also the sequences $\left\{\left(1-\left|z_{n}\right|^{2}\right) \varphi_{i}^{\prime}\left(z_{n}\right)\right\}$ converge.

Proof. The implication $(1) \Rightarrow(2)$ is trivial.

We prove the implication $(2) \Rightarrow(3)$. Let $\left\{z_{n}\right\} \in \Delta$. By permuting the index set, if necessary, we can suppose that

$$
\{1, \ldots, N\}=J_{1}\left\{z_{n}\right\}+\cdots+J_{p}\left\{z_{n}\right\}
$$

where $1 \leq p \leq N$. For $j=1, \ldots, p$, write $a_{j}=\lim _{n \rightarrow \infty} \varphi_{j}\left(z_{n}\right)$ and

$$
\Lambda_{j}=\lim _{n \rightarrow \infty}\left(1-\left|z_{n}\right|^{2}\right) \sum_{i \in J_{j}\left\{z_{n}\right\}} \lambda_{i} \varphi_{i}^{\prime}\left(z_{n}\right) .
$$

We aim to show that $\Lambda_{j}=0$ for each $j$. We have, for $k=1, \ldots, N$,

$$
\begin{aligned}
\left(1-\left|z_{n}\right|^{2}\right)\left(T z^{k}\right)^{\prime}\left(z_{n}\right) & =k\left(1-\left|z_{n}\right|^{2}\right) \sum_{i=1}^{N} \lambda_{i} \varphi_{i}\left(z_{n}\right)^{k-1} \varphi_{i}^{\prime}\left(z_{n}\right) \\
& \rightarrow k \sum_{j=1}^{p} a_{j}^{k-1} \Lambda_{j} \quad \text { as } n \rightarrow \infty .
\end{aligned}
$$


Since $T z^{k} \in \mathcal{B}_{0}$ by assumption, these limits must vanish. Hence

$$
\left(\begin{array}{cccc}
1 & 1 & \cdots & 1 \\
a_{1} & a_{2} & \cdots & a_{p} \\
a_{1}^{2} & a_{2}^{2} & \cdots & a_{p}^{2} \\
\vdots & \vdots & & \vdots \\
a_{1}^{p-1} & a_{2}^{p-1} & \cdots & a_{p}^{p-1}
\end{array}\right)\left(\begin{array}{c}
\Lambda_{1} \\
\Lambda_{2} \\
\Lambda_{3} \\
\vdots \\
\Lambda_{p}
\end{array}\right)=\left(\begin{array}{c}
0 \\
0 \\
0 \\
\vdots \\
0
\end{array}\right) .
$$

But the determinant of this Vandermonde matrix equals $\prod_{1 \leq j<k \leq p}\left(a_{j}-a_{k}\right)$, which by the definition of the $a_{j}$ 's is nonzero. Hence the matrix is invertible and we deduce that $\Lambda_{1}=\cdots=\Lambda_{p}=0$. This completes the proof of (3).

Finally we show the implication $(3) \Rightarrow(1)$. By calculating as in (5.1), we can check that $T z^{k} \in \mathcal{B}_{0}$ for any positive integer $k$. Since the set of all analytic polynomials is dense in $\mathcal{B}_{0}$, we get (1).

5.2. Remark. The number $N$ in condition (2) of Theorem 5.1 is the best possible: it is not sufficient to require $T z^{k} \in \mathcal{B}_{0}$ for any smaller number of monomials. For instance, in the case $N=2$ consider the following trivial example: Let $\varphi_{1}=\sigma$ and $\varphi_{2}=-\sigma$ where $\sigma(z)=\exp ((z+1) /(z-1))$ is the standard singular inner function, which is not a member of $\mathcal{B}_{0}$. Then $\left(C_{\varphi_{1}}+C_{\varphi_{2}}\right) z=0 \in \mathcal{B}_{0}$ but $\left(C_{\varphi_{1}}+C_{\varphi_{2}}\right) z^{2}=2 \sigma^{2} \notin \mathcal{B}_{0}$.

Next we study the compactness of $T$ as an operator on $\mathcal{B}_{0}$.

5.3. Theorem. The following are equivalent:

(1) The operator $T$ is compact from $\mathcal{B}_{0}$ into $\mathcal{B}_{0}$.

(2) $\sum_{i \in I_{j}\left\{z_{n}\right\}} \lambda_{i} \varphi_{i}^{\#}\left(z_{n}\right) \rightarrow 0$ as $n \rightarrow \infty$ for all $\left\{z_{n}\right\} \in \Delta, j=1, \ldots, N$.

(3) $\sum_{i \in I_{j}^{*}\left\{z_{n}\right\}} \lambda_{i}=0$ for all $\left\{z_{n}\right\} \in \Delta, j=1, \ldots, N$.

(4) $\sum_{i \in I_{j}^{\#}\left\{z_{n}\right\}} \lambda_{i}=0$ for all $\left\{z_{n}\right\} \in \Delta, j=1, \ldots, N$ with $\varphi_{j}^{\#}\left(z_{n}\right) \not \rightarrow 0$.

5.4. Remark. The only difference to Theorem 3.2 is that the conditions (2)-(4) are imposed for all indices $j=1, \ldots, N$ instead of only $j \in I\left\{z_{n}\right\}$. These stronger conditions imply that $T$ maps $\mathcal{B}_{0}$ into $\mathcal{B}_{0}$, so this is not needed to be assumed a priori. In fact, if we assume that $T\left(\mathcal{B}_{0}\right) \subset \mathcal{B}_{0}$ (i.e. the conditions of Theorem 5.1 hold), then the compactness of $T$ on $\mathcal{B}_{0}$ is equivalent to its compactness on $\mathcal{B}$, and Theorem 3.2 applies. This can be seen by examining the function-theoretic characterizations given above, but also follows more abstractly from the fact that the inclusion $\mathcal{B}_{0} \subset \mathcal{B}$ corresponds to the canonical embedding of $\mathcal{B}_{0}$ into its bidual (see $[1,14]$ ) and, as a consequence, $T: \mathcal{B} \rightarrow \mathcal{B}$ can be identified with the biadjoint of its restriction $\mathcal{B}_{0} \rightarrow \mathcal{B}_{0}$.

We will use the following well-known criterion for compact sets in $\mathcal{B}_{0}$ (see e.g. [12]). 
5.5. Lemma. A bounded set $E$ in $\mathcal{B}_{0}$ is relatively compact if and only if

$$
\lim _{|z| \rightarrow 1} \sup \left\{\left(1-|z|^{2}\right)\left|f^{\prime}(z)\right|: f \in E\right\}=0 .
$$

Proof of Theorem 5.3. The proof is almost identical to that of Theorem 3.2, and we just briefly indicate the required modifications.

To prove $(1) \Rightarrow(2)$, we let $\left\{z_{n}\right\} \in \Delta$ and assume that $j=1$. We consider test functions $f_{n}$ of the form

$$
f_{n}(z)=\alpha_{\varphi_{1}\left(z_{n}\right)}(z) \prod_{i \notin I_{1}\left\{z_{n}\right\}} \alpha_{\varphi_{i}\left(z_{n}\right)}(z)^{2}-\gamma_{n} .
$$

Now we do not know if $\left\{f_{n}\right\}$ converges to zero, but we may apply Lemma 5.5 to the set $\left\{T f_{n}\right\}$, which by assumption is relatively compact. This yields that $\left(1-\left|z_{n}\right|^{2}\right)\left|\left(T f_{n}\right)^{\prime}\left(z_{n}\right)\right| \rightarrow 0$ as $n \rightarrow \infty$, and the rest of the proof follows easily.

To prove $(2) \Rightarrow(1)$, we first observe that (2) ensures that $T\left(\mathcal{B}_{0}\right) \subset \mathcal{B}_{0}$. Indeed, for $\left\{z_{n}\right\} \in \Delta$ and $j=1, \ldots, N$, we have

$$
\begin{aligned}
\lim _{n \rightarrow \infty} & \left(1-\left|z_{n}\right|^{2}\right) \sum_{i \in I_{j}\left\{z_{n}\right\}} \lambda_{i} \varphi_{i}^{\prime}\left(z_{n}\right) \\
= & \lim _{n \rightarrow \infty} \sum_{i \in I_{j}\left\{z_{n}\right\}} \lambda_{i}\left(1-\left|\varphi_{i}\left(z_{n}\right)\right|^{2}\right) \varphi_{i}^{\#}\left(z_{n}\right) \\
& =\lim _{n \rightarrow \infty}\left(1-\left|\varphi_{j}\left(z_{n}\right)\right|^{2}\right) \sum_{i \in I_{j}\left\{z_{n}\right\}} \lambda_{i} \varphi_{i}^{\#}\left(z_{n}\right)=0 .
\end{aligned}
$$

Since the sets $I_{j}\left\{z_{n}\right\}$ induce a subpartition of the sets $J_{j}\left\{z_{n}\right\}$, Theorem 5.1 shows that $T\left(\mathcal{B}_{0}\right) \subset \mathcal{B}_{0}$. The compactness of $T$ is now immediate because condition (2) clearly implies the corresponding condition of Theorem 3.2 and so $T$ is compact even on the space $\mathcal{B}$.

Finally, the equivalence of conditions (2)-(4) is established exactly as before: the arguments used in the proof of Theorem 3.2 did not really depend on the assumption $j \in I\left\{z_{n}\right\}$.

Acknowledgement. This research was initiated when the second author was visiting Nippon Institute of Technology and Niigata University in Japan in 2007. He is very grateful to Professors Kei Ji Izuchi and Shûichi Ohno for hospitality and financial support. He also wishes to acknowledge the hospitality and pleasant atmosphere of the Department of Mathematics at the University of Zaragoza in Spain during his stay in 2008.

\section{REFERENCES}

1. J.M. Anderson, J. Clunie and Ch. Pommerenke, On Bloch functions and normal functions, J. Reine Angew. Math. 270 (1974), 12-37.

2. C.C. Cowen and B.D. MacCluer, Composition Operators on Spaces of Analytic Functions, CRC Press, Boca Raton, 1995.

3. J.B. Garnett, Bounded Analytic Functions, Academic Press, New York, 1981; rev. ed. by Springer, New York, 2007. 
4. P. Ghatage and D. Zheng, Hyperbolic derivatives and generalized Schwarz-Pick estimates, Proc. Amer. Math. Soc. 132 (2004), 3309-3318.

5. P. Gorkin and R. Mortini, Norms and essential norms of linear combinations of endomorphisms, Trans. Amer. Math. Soc. 358 (2006), 553-571.

6. H. Hedenmalm, B. Korenblum and K. Zhu, Theory of Bergman Spaces, Springer, New York, 2000.

7. T. Hosokawa, Differences of weighted composition operators on the Bloch spaces, Complex Anal. Oper. Theory (to appear).

8. T. Hosokawa and S. Ohno, Topological structure of the sets of composition operators on the Bloch spaces, J. Math. Anal. Appl. 314 (2006), 736-748.

9. T. Hosokawa and S. Ohno, Differences of composition operators on the Bloch spaces, J. Operator Theory $\mathbf{5 7}$ (2007), 229-242.

10. K.J. Izuchi and S. Ohno, Linear combinations of composition operators on $H^{\infty}$, J. Math. Anal. Appl. 338 (2008), 820-839.

11. T. Kriete and J. Moorhouse, Linear relations in the Calkin algebra for composition operators, Trans. Amer. Math. Soc. 359 (2007), 2915-2944.

12. K. Madigan and A. Matheson, Compact composition operators on the Bloch space, Trans. Amer. Math. Soc. 347 (1995), 2679-2687.

13. P.J. Nieminen, Compact differences of composition operators on Bloch and Lipschitz spaces, Comput. Methods Funct. Theory 7 (2007), 325-344.

14. K. Zhu, Operator Theory in Function Spaces, Dekker, New York, 1990; 2nd ed. by Amer. Math. Soc., Providence, 2007.

Institute of Basic Science, Korea University, Seoul 136-713, Korea

E-mail address: turtlemumu@yahoo.co.jp

Department of Mathematics and Statistics, University of Helsinki, PO Box 68, FI-00014 HELSINKI, FinLAND

E-mail address: pjniemin@cc.helsinki.fi

Nippon Institute of Technology, Miyashiro, Minami-Saitama 345-8501, Japan

E-mail address: ohno@nit.ac.jp 